\title{
LINK MARGIN FOR WIRELESS RADIO COMMUNICATION LINK
}

\author{
Kamaljeet Singh, A.V. Nirmal and S.V. Sharma \\ Systems Engineering Group, ISRO Satellite Centre, Bangalore, India
}

\begin{abstract}
The systematic evaluation of the link budget calculation for the satellite and terrestrial communication is presented in this article. Communication link between the satellite and earth station is dependent on various propagation and associated losses which are either constant or vary with weather conditions. Role of receiver noise, antenna pointing mechanism, atmospheric effects, slant height, interferences, bit error rate on the link margin are detailed in this article. Various equations for link budget calculation and a comparative table at various frequency bands are shown in this article which is useful for predicting link margin of LEO, GEO and Deep space missions. Tele-command, telemetry and ranging link margin at various frequencies are presented and budget analysis at Ka-band frequency performed.
\end{abstract}

\section{Keywords:}

Link Budget, Margin, Atmospheric Effects, Noise, Propagation Losses, Uplink, Downlink

\section{INTRODUCTION}

Radio communication link can be established by various channels such as wires, coaxial cables, fibre optic cables, waveguide, atmosphere, empty space. Radio communication through wireless systems are preferred over the wired system for long range communication as the signal power in wired communication system attenuates exponentially with distance compared to space decay resulting in reduced signal strength. Wireless communication involves transfer of information in space using sound, optical, infrared, radio frequency techniques and most widely employed is the radio frequency communication as it offers wider bandwidth and can penetrate fog, foliage, dust, buildings and vehicles also. Connectivity for voice, video and data communications can be provided using wireless systems such as Wireless Local Area Networks (WLANs), Direct Broadcast Satellite (DBS) television service, paging systems, Global Positioning Satellite (GPS) service, Radio Frequency Identification (RFID) systems. Present day systems employ higher frequencies to avoid spectrum crowding as well as offering wider bandwidth [1].

Wireless systems can be categorized as ground or satellite based system and capable of providing multipoint-to-multipoint systems having full duplex capability for allowing simultaneous communication between the users. Satellite networks can provide wide area coverage and adopted in broadcasting internet, earth observation and military communications. In a satellite link delay from earth to satellite to earth is about $240 \mathrm{~ms}$ while in terrestrial link it will be far less due to placement of receiver on tall buildings or hilltops at certain distances. Very high bandwidths and very high data rates are achievable in a satellite based communication system at higher frequency ranges at the expense of vulnerability associated with the weather conditions.
Major advantage of Satellite base link is minimal requirement of re-transmitters as are required in the terrestrial link. Transmission of signals over a satellite communication link requires Line-of-Sight $(L o S)$ communication, but since theoretically three equidistant satellites in the geosynchronous orbit can effectively cover over 90 percent of the earth surface, the need for multiple re-transmissions is removed. Satellite based systems can be classified into Geostationary (GEO) satellites and Non-Geostationary (NGEO) satellites and NGEO can further be segregated as Low earth orbit (LEO) and Medium-altitude orbit (MEO) systems according to the satellite position. The satellite situated at $500<h<2000 \mathrm{~km}$ are LEO satellites and the satellites with $5000<h<20000 \mathrm{~km}$ are MEO satellites and the satellites with $h=35800 \mathrm{~km}$ are GEO satellites. Since the satellite footprint decreases in size as the orbit becomes lower, LEO and MEO satellite systems require larger constellations than the GEO satellites in order to achieve the global coverage and avoid data transmission delays. Satellites in LEO are not affected by the Sun outages and satellite eclipses [2]. LEO satellites are used for personal communication purpose as it produces effective signal strength with less power consumption and minimum losses due to vicinity to the Earth but needs multiple satellites for full coverage. Due to free space attenuation and large round trip latency, GEO based system covers wider area but needs high transmitter power and accurate predictions to overcome interferences for maintaining reliable link [3]. The LEO can be further sub-divided into big LEO and little LEO categories. The big LEOs can offer voice, fax, telex, paging and data services whereas the little LEOs can provide only data services either real time services or store and forward services.

Link budget is figure of merit for an effective and reliable link between receiver and transmitter for terrestrial as well as satellite based communication link. Main parameters for satellite communication link are frequency of operation, range requirement, antenna gain of transmitter and receiver antenna, data rate, receiver bandwidth, noise figure and system losses. At millimetre wave frequencies more bandwidth is available and antennas are having higher gain but weather conditions adversely affect the link as atmospheric absorption is much more and can attenuate signal at much higher rate. Present days system are replacing conventional NRZ signalling by PAM4, a modulation scheme that takes half the bandwidth to transmit the same payload as the equivalent NRZ signal. In this article satellite based link budget calculation is detailed at various frequency bands covering various orbits. Also the loss mechanism associated with terrestrial link and satellite based communication link is explained as compared to terrestrial link the main factors contributing the signal losses are free space loss, rain, oxygen and antenna misalignment [4]. Further, margin calculation in Ka-band is taken as a case study as $5 \mathrm{G}$ communications in the future will be having spectrum in the $28 \mathrm{GHz}, 37 \mathrm{GHz}$ and $39 \mathrm{GHz}$ frequency bands. 


\section{RADIO FREQUENCY COMMUNICATION LINK}

Received power is one of the most important factors for having a reliable radio communication link (Fig.1). The received power by a radio antenna having circular aperture antenna of diameter $D$ is given by Friis radio link formula as,

$$
P_{r}=\frac{P_{t} G_{t} A_{e}}{4 \pi R^{2}}=\frac{G_{t} G_{r} \lambda^{2}}{(4 \pi R)^{2}} P_{t}=W \eta\left(\frac{\pi \cdot D}{\lambda}\right)^{2}
$$

where,

$$
\begin{aligned}
& P_{t} G_{t}=E I R P \\
& \left(\frac{\lambda}{4 \pi R}\right)^{2}=F S L \\
& A_{e}=\eta \frac{\pi D^{2}}{4}=\frac{7160}{f^{2}(M H z)}, \\
& G_{r}=\frac{4 \pi A_{e}}{\lambda^{2}} \text { and in dB terms }
\end{aligned}
$$$$
P_{r}(d B W)=E I R P+G_{r}-L_{P}
$$

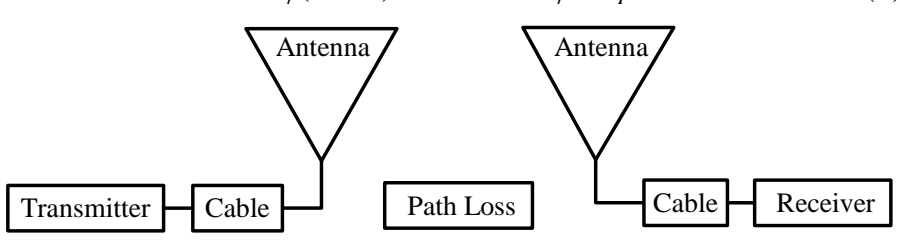

Fig.1. Transmitter-receiver architecture for radio communication link

Alternatively, power received by an isotropic antenna by the known electric field strength $E$ at the receiver is given as,

$$
P_{r}(d B m)=E\left(d B \frac{\mu V}{m}\right)-20 \log f_{M H z}-77.2
$$

The received power $P_{r}$ is referred as carrier power $(C)$ and is independent of the modulation scheme. Main functions of radio receiver are high gain, selectivity, down conversion, detection and transmitter isolation The calculation of link budget involves calculation of many parameters such as effective isotropic radiated power (EIRP), free space loss (FSL), carrier to noise ratio, bit error rate, various losses such as impedance and polarization mismatch of antennas, propagation effects and multipath effects to be taken into account apart from received power. Quality of a radio-frequency communication link is a function of various parameters such as receiver sensitivity, background noise level in the band, transmitted signal power level, transmitting/receiving antenna polarization as well as gain, dissipation or propagation losses and implementation losses [5]. The Table. 1 shows the comparison between the terrestrial and satellite based communication systems.

Link budget needs the following information's:

- Latitude and longitude of the uplink and downlink earth stations.

- Planned data or information rate.

- Modulation type (BPSK or QPSK)
- Forward error correction rate (1/2 or $3 / 4)$

- Spread Factor - if any (use only for spread spectrum systems)

- Uplink and Downlink frequencies.

- Uplink and Downlink antenna sizes.

- Uplink and Downlink antenna efficiency.

- Uplink and Downlink transmit and receive gains at frequency.

- Minimum digital signal strength $\left(E_{b} / N_{o}\right)$ for desired Bit Error Rate (BER) performance.

Table.1. Comparison between terrestrial and satellite communication

\begin{tabular}{|c|c|c|}
\hline Parameters & $\begin{array}{c}\text { Terrestrial } \\
\text { Communication }\end{array}$ & $\begin{array}{c}\text { Satellite } \\
\text { Communication }\end{array}$ \\
\hline Frequency & L-band & C-, Ku-, Ka-band \\
\hline $\begin{array}{c}\text { Atmospheric } \\
\text { effects/Rain attenuation }\end{array}$ & Negligible & $\begin{array}{c}\text { High (changes } \\
\text { with rain rate) }\end{array}$ \\
\hline Material penetration & Better & Not good \\
\hline Antenna Diameter & Larger & Smaller \\
\hline Spectrum bandwidth & Lower & Higher \\
\hline Beam width & Higher & Smaller \\
\hline Link Loss & Lower & $\begin{array}{c}\text { Higher }(6 \text { dB for } \\
\text { twice the freq. }\end{array}$ \\
\hline Point-to-point services & Not good & Better \\
\hline System Configuration & Simple & Complex \\
\hline Delay time & Less & More \\
\hline Antenna Configuration & $\begin{array}{c}\text { Same at Rx/Tx } \\
\text { end }\end{array}$ & Different antennas \\
\hline Fade Margin & Less & More \\
\hline
\end{tabular}

\section{RADIO PROPAGATION LOSSES}

In free space waves travel in straight lines but in the presence of earth and its atmosphere (an altitude of $20 \mathrm{~km}$ ) the path of the wave gets altered. Radio propagation consist of mainly three electromagnetic waves namely surface waves, sky waves and space waves. Wave propagation in high frequency region is mainly surface and sky whereas above it the propagation is primarily space waves. During radio propagation electromagnetic waves suffer several atmospheric effects that results in power loss and these effects are: Reflection and refraction, diffraction and scattering. These effects results in the large scale or small scale fading in accordance with the size of the object compared to the wavelength. In case of satellite based system the main losses are categorized are: Free space losses, Atmospheric losses Pointing losses and Inter symbol interferences. These losses are detailed as below:

\subsection{FREE SPACE LOSSES}

Free space loss is the dominant component in the loss of the strength of the signal. As the radio signal travels through space, it deteriorates for two reasons: 
1. The signal spreads out in space, inversely proportional to the square of the distance.

2. Absorption by the atmosphere and higher the frequency, the greater the attenuation.

$$
-L=C+20 \times \log (D)+20 \times \log (F)
$$

where, $D$ is the distance $(\mathrm{kms}), F$ is the frequency in $\mathrm{MHz}$ and the constant $C$ is 32.5

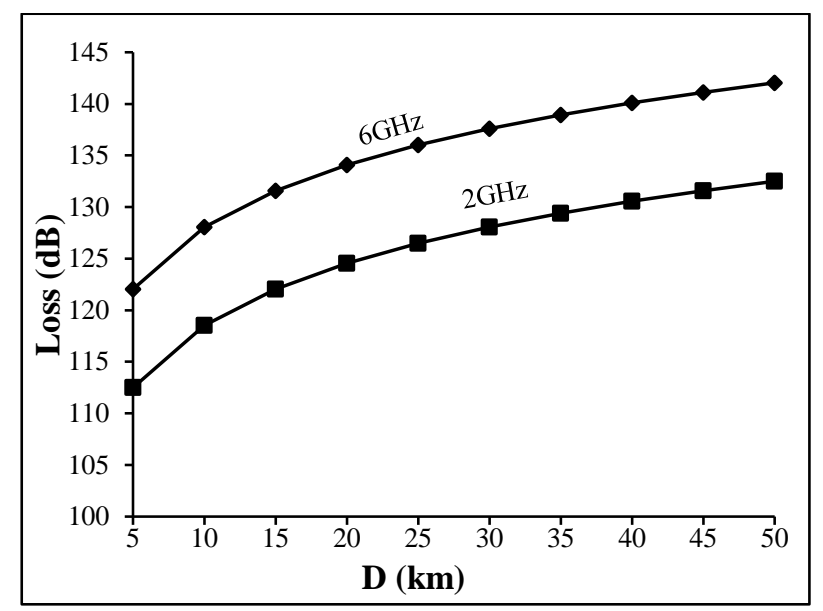

Fig.2. Free space path loss with distance

The plot in Fig. 2 shows that at higher frequencies path loss is more with the distance compared to lower end of the frequency which is evident from Eq.(3).

\subsection{ATMOSPHERIC LOSSES}

Atmosphere losses can be categorized as attenuation or absorption where absorption phenomena arise in clear sky while attenuation is due to weather. Atmospheric effects in satellite transmissions increases with the increase of frequency and the effects of the atmosphere in signal attenuation become higher for frequencies above $10 \mathrm{GHz}$.

\subsection{POINTING LOSSES}

The pointing losses are due to off-axis losses arise from the satellite and ground station. The misalignment of the antenna resulted in the loss of $1 \mathrm{~dB}$.

\subsection{IONOSPHERIC EFFECTS}

All radio waves transmitted by satellites to the earth or vice versa must pass through the ionosphere containing ionized particles and these effects in satellite communication give rise to polarization rotation and the scintillation which decreases usually with the increase of the square of the frequency. Further refractive index causes scattering and multipath effects in the signal and is detected as variations in amplitude, phase, polarization, angle of arrival of the radio waves. The effect of atmospheric scintillation effect is taken into account in link margin as fade margin.

\subsection{TROPOSPHERIC EFFECTS}

Radio waves passing through troposphere are scattered, depolarized, absorbed and attenuated due to hail, raindrops or other atmospheric gases. Heavy rains cause severe attenuation of the signal as well as drops of rain also add to depolarization. The rain rate attenuation measured in $\mathrm{dB}$ is expressed below:

$$
\text { Attenuation }_{(0.01)}=k \cdot R R^{\alpha} d r(\mathrm{~dB})
$$

where, $R R=99.9 \%$ rain rate for the rain region ( $\mathrm{mm} / \mathrm{hour}), k R R^{\alpha}$ $=$ specific rain attenuation $(\mathrm{dB} / \mathrm{km}), d=$ link distance $(\mathrm{km}), r=$ $1 /\left[1+\left(d / d_{o}\right)\right]=(0.998), d_{o}=$ Effective Path Length $(\mathrm{km})=35 \mathrm{e}^{-}$ $0.015 R R$. Rain margin for $\mathrm{Ku}$-band is typically $99.6 \%$ and $\mathrm{C}$-band is $99.96 \%$. The heavy rain causes severe attenuation compared to drops of rain as shown in Table. 2 and present problem at more than $10 \mathrm{GHz}$.

Table.2. Typical effect of rain attenuation

\begin{tabular}{|c|c|}
\hline Conditions & Range \\
\hline Dry Air & $75 \mathrm{~km}$ \\
\hline Light Drizzle & $68 \mathrm{~km}$ \\
\hline Light Rain & $20 \mathrm{~km}$ \\
\hline Heavy Rain & $5 \mathrm{~km}$ \\
\hline
\end{tabular}

Multipath fading results from varied refractive index gradient with atmospheric height. The probability of predicting the fading $\left(p_{w}\right)$ exceeding small time $\%$ is,

$$
p_{w}=K d^{3}\left(1+\left|\varepsilon_{p}\right|\right)^{-1.2} \times 10^{-0.033 f-0.001 h_{L}-A / 10}
$$

where, $d$ is the path length, $f$ is the frequency in $\mathrm{GHz}, \varepsilon_{p}$ is the slope in milli-radians, $K$ is radio climatic factor taking into account location, A is fade depth and $h_{L}$ height of the lower of two terminals. Increase in fade depth $A$ of $10 \mathrm{~dB}$, the probability of link reduction reduces by a factor of 10 . If an availability of $99.99 \%$ is required, an extra $10 \mathrm{~dB}$ fade margin will be needed compared with an availability of 99.95 .

Fade margin at Ka-band is taken as $14 \mathrm{~dB}$ for $99.9 \%$ availability. De-polarization may occur when an orthogonal component is created due to the passing of the signal through the ionosphere. It can be caused by the ice layer also which is on the top of each rain area. There are two ways to measure its effect, cross-polarization discrimination and polarization isolation. As radio waves cross troposphere, radio frequency energy will be converted into thermal energy due to absorption and that attenuates signal. Absorption is caused by the presence of oxygen and water vapour molecules. Gas absorption will cause loss of signal which can be given as,

$$
A_{G}(\mathrm{~dB})=\operatorname{Labs} \mid 90^{\circ}(\mathrm{dB} / \mathrm{km}) \operatorname{cosec} \theta . T_{\text {trop }}(\mathrm{km})
$$

Other atmospheric gases only become a problem in very dry air conditions above $70 \mathrm{GHz}$. Thereby, losses caused by atmospheric absorption vary with frequency and not significant with an elevation angle of $5^{\circ}$ or higher and for frequencies below $10 \mathrm{GHz}$. Conversion from peak rain rate to the loss on a given link can be calculated as:

- Determine the peak rain rate

- Determine the loss per kilometre for the frequency and polarization

- Determine effective path length for the path length and rain rate

- Determine the peak link loss by multiplying the effective path length by loss per kilometre 


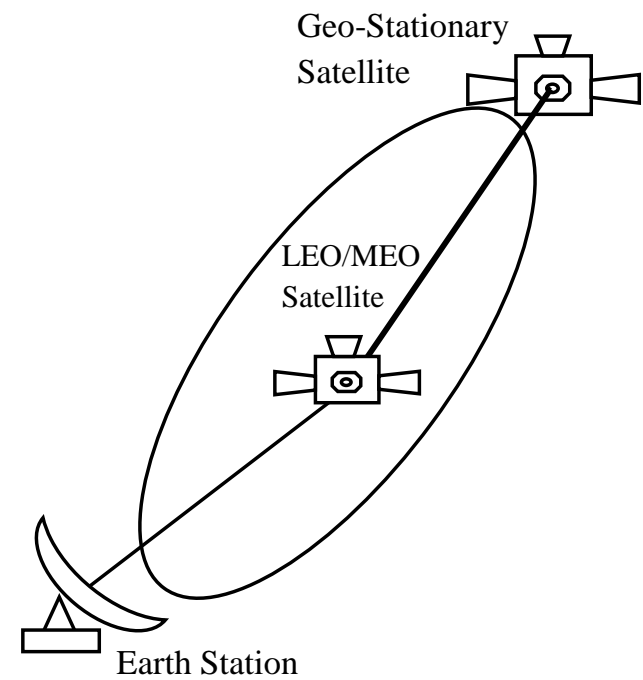

Fig.4. Interference of GEO link due to LEO/MEO satellite

Interference mitigation techniques include:

- Spatial isolation/Satellite diversity

- Time/frequency/time isolation

- Satellite antenna side-lobes/Earth station antenna side-lobes

- Band planning

- Co-coverage avoidance

- Minimum look angle restrictions between earth terminals and terrestrial fixed service links

Table.3. Propagation Losses associated with Radio Signal

\begin{tabular}{|c|l|l|}
\hline \multicolumn{2}{|c|}{ Free Space Loss } \\
\hline \multirow{4}{*}{$\begin{array}{c}\text { Atmospheric } \\
\text { Losses }\end{array}$} & \multirow{2}{*}{\begin{tabular}{l} 
Ionospheric Effects \\
\cline { 3 - 3 }
\end{tabular}} & Faraday Rotation \\
\cline { 3 - 3 } & Tropospheric Effects & Scintillation Effects \\
\cline { 3 - 3 } & Gas Absorption \\
\cline { 3 - 3 } & Sky Noise \\
\cline { 3 - 3 } $\begin{array}{c}\text { Pointing } \\
\text { Losses }\end{array}$ & & Depolarization \\
\hline
\end{tabular}

In satellite based system, satellite EIRP and bandwidth are important parameters and to be considered critically at the design stage.

\subsection{RECEIVER SENSITIVITY}

Receive sensitivity is dependent on the noise figure and required signal-to-noise ratio of the system. System noise comprises of the low noise amplifier (LNA) generated noise and associated noises in terms of noise temperature contributes the overall noise along with elevation angle of the antenna system above horizon. The required signal-to-noise ratio is dependent on the modulation technique. Typically, the higher the data rate of the system, the more bandwidth is needed. This means the receiver must capture more signal, which means more noise is captured. The minimum required Rx sensitivity $\left(P_{\min }[\mathrm{dBW}]\right)$ can be calculated from the minimal required receiver noise input power $\left(P_{n}[\mathrm{dBW}]\right)$ and SNR,

$$
\begin{gathered}
P_{\min }=P_{n}+S N R \\
P_{n}=F+10 \cdot \log _{10}\left(k T_{0} B\right)
\end{gathered}
$$

where, $F$ is the receiver noise figure $(6 \mathrm{~dB}), K$ is Boltzmann's constant $\left(1.38 .10^{-23} \mathrm{Ws} / \mathrm{K}\right), T_{0}$ the absolute temperature $(290 \mathrm{~K})$ and $B$ the receiver noise bandwidth $[\mathrm{Hz}]$

Link performance can be affected by other factors such as Human error and equipment failure, Crossbow Leakage, Adjacent satellites, Terrestrial services, deliberate interference. In case of satellite link design, interference may be considered as a form of noise and hence, system performance is determined by the ratio of wanted to interfering powers.

\subsection{NOISE CONCEPT}

Noise can be classified as thermal, galaxy, atmospheric, switching transistor, inter-modulation, interfering, LO phase noise and is represented in terms of noise temperature. Noise temperature provides a way of determining minimum detectable signal in receiver and generation of thermal noise in active and passive devices of the receiving system .The total noise power at the output of the receiver is due to the contributions from antenna pattern, antenna loss, transmission loss and from the receiver components. The noise power is given by the Nyquist equation as,

$$
N=k T_{0} B
$$

where, $P_{n}$-delivered to load with matched impedance to source noise; $k$ - Boltzmann constant $=1.39 \times 10^{-23}$ joules $/ \mathrm{K}=-228.6$ $\mathrm{dBW} / \mathrm{K} / \mathrm{Hz} ; T_{0}$ - Noise temperature of source in Kelvin; $B$ - Noise bandwidth in which the temperature is measured in $\mathrm{Hz}$.

A noisy component is characterized by the noise figure $(F)$ which measures the degradation of signal-to-noise ratio between the input and output of the component. Relation between equivalent noise temperature and noise figure is given as,

$$
T_{e}=(F-1) T_{0}
$$

Performance of the receiving system is characterized by the system noise temperature and desirable to be kept low. Overall noise contribution is combination from the internal system as well as from external sources which affects the $G / T$ ratio. Quality of the signal in the reception is dependent on $T$ and major contribution came from sky noise which is a combination of galactic and atmospheric effects. Galactic effects are due to the addition of the cosmic background radiation and the noise temperature of radio stars, galaxies, nebulae and decreases with the increase of frequency. This value is quite low and taken as $3 \mathrm{~K}$ towards zenith and $50 \mathrm{~K}$ towards horizon. Elevation angle also affects the overall noise temperature and the value increases as the elevation angle is reduced below 10 [1]. In case of sun outages, noise temperature of the system becomes extremely high resulting in link loss. The scenario is also repeated at satellite eclipse due to thermal vibrations due to satellite crossing the cone of shadow of the Earth.

\subsection{SATELLITE COMMUNICATION LINK}

The elements of satellite systems are earth segment and space segment. Earth segment comprises of a network of transmit and 
receive earth stations whereas space segment comprises of a satellite [6-7]. The performance objectives of the satellite link is specified in terms of allowable $(S / N)$ or BER for a given signal or as a minimum allowable carrier to noise power ratio $C / N$. Reliable link in satellite communication can be made by ensuring minimum signal-to-noise ratio $(S / N)$ in the receiver baseband channel, optimizing transmitter power and RF bandwidth. The $S / N$ ratio is dependent on the carrier-to-noise ratio $(C / N)$ of the $\mathrm{RF}$ or IF signal in the receiver, modulation type and the RF/IF bandwidth in the receiver. The carrier power $(C)$ and the noise power in the earth station receiver $(N)$ can be represented as Eq.(1) and Eq.(7):

$$
\frac{C}{N}=\frac{P_{r}}{N}=\frac{P_{t} G_{t} G_{r}}{(4 \pi R / \lambda)^{2}} / k T_{0} B
$$

The above value is specified in terms of percentage of outage time and at $\mathrm{C}$-band it is taken as $0.01 \%$ of a year, at $\mathrm{Ku}$-band it is $0.1 \%$ of a year and for Ka-band $0.2 \%$ of a year.

$$
\begin{gathered}
\frac{C}{N}=\frac{P_{t} G_{t}}{k B}\left[\frac{\lambda}{4 \pi R}\right]^{2} \frac{G_{r}}{T_{0}} \\
\text { So }(C / N) \alpha\left(G_{r} / T_{0}\right)
\end{gathered}
$$

The ratio of $G / T$ is known as figure of merit and is dependent on frequency and noise temperature. The noise generated in the receiver systems depends on the system noise temperature and the bandwidth used for communication. In case of downlink,

$$
\begin{gathered}
G / T(\mathrm{~dB} / \mathrm{K})=\text { Receiver Antenna gain }-10 \log (\text { system noise } \\
\text { temperature })(\mathrm{dB} / \mathrm{K})
\end{gathered}
$$

System noise temperature can be shown as (antenna is followed by line and receiver)

$$
\begin{aligned}
T_{\text {sys }} & =T_{\text {ant }}+T_{\text {cas }}=T_{\text {ant }}+T_{1}+\frac{T_{2}}{G_{1}}+\frac{T_{3}}{G_{1} G_{2}} \\
& =\eta_{\text {rad }} T_{A}+\left(L_{F}-1\right) 290+T_{L N A}\left(L_{F} / G_{R}\right)
\end{aligned}
$$

Taking fixed LNA temperature of $100 \mathrm{~K}$ with the elevation angle of $30^{\circ}$, the Table. 4 provide the comparison of gain with the antenna size $(\eta=55 \%)$ at $4 \mathrm{GHz}[8]$

Table.4. Effect of antenna diameter on $G / T$

\begin{tabular}{|c|c|c|c|}
\hline $\begin{array}{c}\text { Antenna Diameter } \\
(\mathbf{f t})\end{array}$ & $\begin{array}{c}\text { Gain } \\
(\mathbf{d B i})\end{array}$ & $\begin{array}{c}\text { G/T } \\
(\mathbf{K})\end{array}$ & $\begin{array}{c}\mathbf{C} / \mathbf{N} \\
(\mathbf{d B})\end{array}$ \\
\hline 6 & 35 & 12 & 4.7 \\
\hline 8 & 37.5 & 15.1 & 7.8 \\
\hline 10 & 39.4 & 17.5 & 10.2 \\
\hline 12 & 41 & 19.4 & 12.1 \\
\hline 14 & 42.3 & 20.8 & 13.5 \\
\hline
\end{tabular}

Gain and beam width of a circular parabolic dish type aperture antenna of diameter $D$ can be approximated as

$$
\begin{gathered}
\text { Antenna Gain }(\mathrm{dBi})=18+20 \log \left(d_{m t r s}\right)+29 \log \left(f_{G H z}\right) ; \\
\text { Beam-width }(\theta \text { in degrees }) \sim \frac{22}{D f} ; \text { Gain }=\frac{16}{\theta^{2}}
\end{gathered}
$$

Noise can be lowered by employing better LNA with low noise figure while keeping antenna gain constant or else employing larger diameter antenna to be used to achieve the same performance. In case of uplink the noise contribution from sky and ground is prominent and changes with elevation angle. Interferences from other earth stations, terrestrial microwave links is considered as noise entering the receiver. Earth station G/T can be written as,

$$
\begin{gathered}
\frac{G}{T}\left(\frac{\mathrm{deg}}{K}\right)=\frac{8 \pi k(Y-1)}{\lambda^{2} S K_{1} K_{2}} \\
K_{1}=K_{g} \cdot K_{r} \cdot K_{d}
\end{gathered}
$$

where, $K_{1}, K_{2}$ are atmospheric and source correction factor.

The values can be calculated for deep space missions using the above equation. Main satellite parameters are orbit, orbit spacing, orbital inclination, constellation size, round trip delay, polarization, beam diameter, transponder bandwidth, antenna gain, antenna diameter, half power beamwidth, EIRP. Satellite communication link can be divided into design of uplink and downlink communication system.

\section{LINK BUDGET CALCULATION}

\subsection{DOWNLINK DESIGN $(C / N)_{D}$}

Downlink parts consist of the portion of link between the satellite and the receiving earth station (Eq.(9)). The downlink design is carried out with the following objectives:

- Minimum $C / N$ at the receiver input to guarantee link continuity

- Carry maximum number of channels

Main criteria to be considered in downlink are the limit on the maximum permissible flux density at the earth's surface so as to avoid interference with the terrestrial links and to maintain $C / N$ ratio above the threshold. $C / N$ ratio can be increased by decreasing the noise bandwidth at the expense of reduction in number of channels.

$$
\left[C / N_{\mathrm{o}}\right]_{D}=[E I R P]_{D}+[G / T]_{G S}-[L]_{D}+228.6(\mathrm{dBHz})
$$

= On-board transmitter EIRP - (path losses + attenuation $)+$ Ground Station $G / T+228.6$

Downlink data is classified as telemetry and ranging data. The telemetry function is the acquisition, conversion, multiplexing, encoding and transmission of the data necessary to establish the status of various spacecraft sub-system and payload data. Ranging data provides the range of the satellite using the on-board transponder facility.

\subsection{UPLINK DESIGN $(C / N)_{U}$}

Uplink parts consist of the portion of link between the transmitting earth station and the satellite (Eq.(10)). The noise contribution from the uplink is less in the overall noise budget and remains constant as noise temperature remains unchanged. At frequencies above $10 \mathrm{GHz}$, propagation disturbances cause variation in received power which can be compensated by employing uplink power control.

$$
\left[C / N_{o}\right]_{U}=[E I R P]_{U}+[G / T]_{U}-[L]_{U}+228.6(\mathrm{dBHz})
$$

$=$ Ground transmitter EIRP $-($ Up link path losses + attenuation $)$ + Satellite $G / T+228.6$ 
Telecommand function is the reception, decoding and distribution of the messages sent from the ground stations to control or change the operational status of the spacecraft main frame and payload systems.

$$
\text { Overall }(C / N)_{T}=(C / N)_{U}+(C / N)_{D}
$$

\subsection{CONCEPT OF BIT ERROR RATE (BER)}

In digital modulation the performance criteria is specified in terms of BER which is the measure of the error probability due to polarity reversal at the time of signal sampling in the receiver. It depends on the noise present and the amount of inter-symbol interference. In case of transmission of polar NRZ waveform, taking $T_{b}$ as the bit period, average energy in each bit can be represented as

$$
E_{b}=P_{r} T_{b}
$$

So for minimum probability of error $\left(N_{0}\right.$ is the noise energy per bit)

$$
B E R=\frac{1}{2} \operatorname{erf} \sqrt{\frac{E_{b}}{N_{0}}}(12) \text { and }\left(\frac{S}{N}\right)_{\max }=\sqrt{\frac{2 E_{b}}{N_{0}}}
$$

Error probability depends inversely on the square root of the bit rate. Using the Eq.(12) for a given BER, the corresponding $E_{b} / N_{0}$ is found out in which implementation margin is added for non-ideal filtering conditions. The relation between $C / N$ and $E_{b} / N_{0}$ can be found out as below:

$$
\begin{aligned}
& \frac{C}{N}=\frac{E_{b}}{N_{0}} \cdot \frac{f_{b}}{B W} \\
& \Rightarrow \frac{C}{N_{0}}(d B)=\frac{E_{b}}{N_{0}}-10 \log \left(\frac{B W}{\text { DataRate }\left(\text { kbps } \times 10^{3}\right)}\right)
\end{aligned}
$$

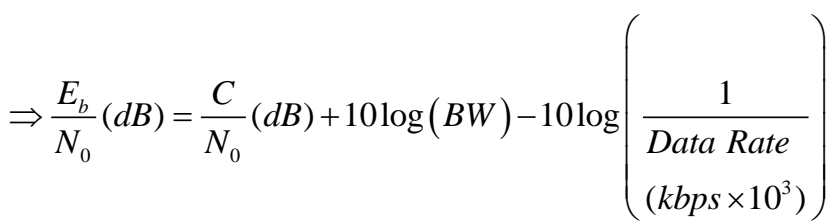

where data rate value are to be kept in $\mathrm{kbps}, f_{b}$ is symbol/data rate and BW is occupied bandwidth. Further symbol rate is written as

$$
f_{b}=\frac{\text { information rate }}{\text { modulation } \times \text { FEC } \times \text { coding }}
$$

Taking information rate as $1544 \mathrm{kbps}$ than for a given modulation type (QPSK=2), feed error correction (FEC) of 0.75 , coding of Reed-Solomon (0.92) resulting in the symbol rate /data rate using Eq.(13) as $1116.9 \mathrm{kHz}$. The error coding mechanism advantage is the introduction of the redundancy to the transmitted data for correcting the errors in the receiver path for further processing [9]. Reed Solomon (R-S) code are one of the error correcting codes belong to the family of non-binary cyclic error correcting codes and are employed for burst error correction. Digital communication system utilizing the RS code is shown in Fig.1 where data gets encoded before modulation and further transmitted via communication channel. The transmitted data having error at the receiver end is decoded at the receiver end with the correction of erroneous symbols. Parameters $\mathrm{C} / \mathrm{N}$ and $\mathrm{Pr} / \mathrm{N}$ are the same for constant envelope signalling (PSK or FSK) but are different for non-constant envelope signalling (ASK, QAM).

\subsection{CONCEPT OF SLANT ANGLE AND COVERAGE}

Slant range is the measurement of the distance of the satellite from the earth station as shown in Fig. 4 and can be calculated using the cosine law as shown:

$$
R_{S}^{2}=D^{2}+R_{E}^{2}-2 D \cdot R_{E} \cos \theta
$$

Slant path suffers less multipath fading compared to horizontal path. The plot between slant range with satellite distance with the elevation angle $(l)$ is plotted in Fig.5.

Coverage angle $(\beta)$ is dependent on elevation angle $(\theta)$ and can be calculated using the Eq.(16).

$$
\frac{R_{E}}{R_{E}+D}=\frac{\cos (\beta+\theta)}{\cos \theta}
$$

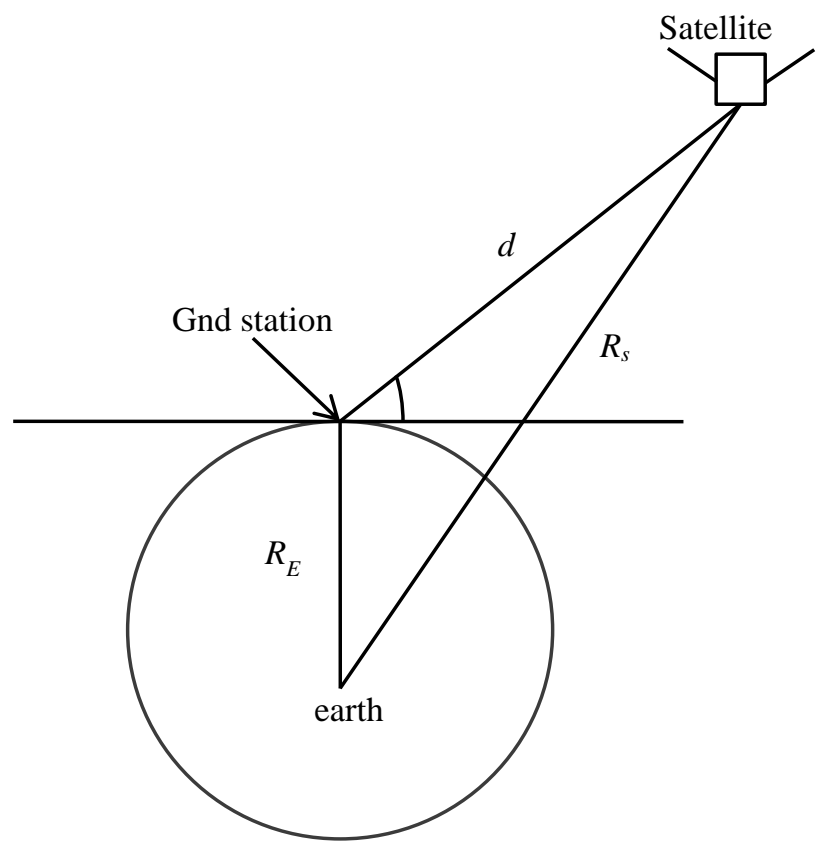

Fig.4. Illustration of the satellite position

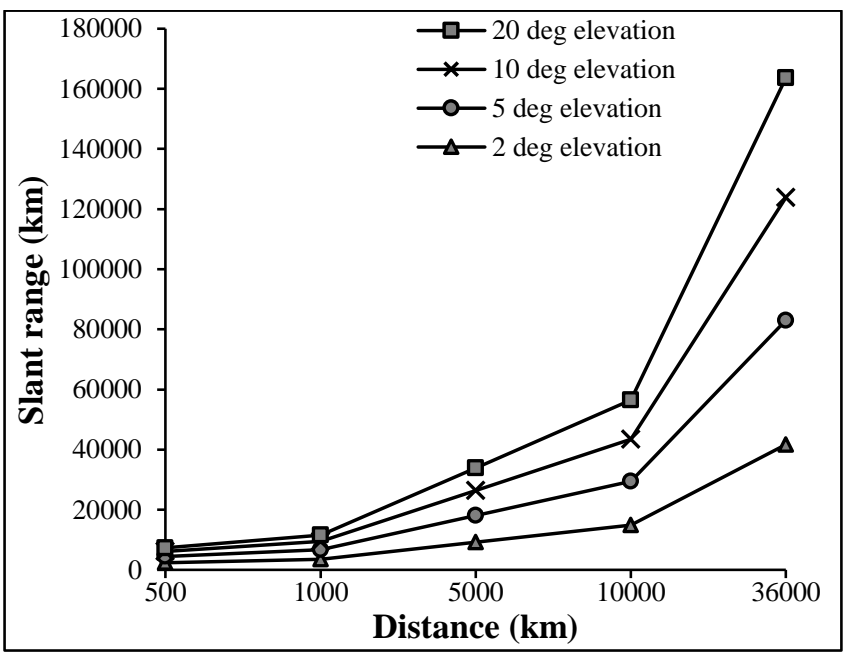

Fig.5. Slant range variation with distance and elevation angle 


\subsection{LINK MARGIN}

The link margin required for the link budget calculations is usually given at the edge of the coverage area to achieve certain $C / N$ at the receiver [10]. It provides account of all the gains and losses from transmitter to receiver via medium [11-12]. It is calculated by taking into account the various losses as shown below:

Sum of all losses $=$ FSL + atmospheric losses + polarization loss + antenna misalignment loss

The main factors responsible for link margin calculation are:

- Transmitting frequency

- Minimum $\mathrm{C} / \mathrm{N}$ requirement

- Type of coverage area

- Nature of obstructions

- Nature of reception (fixed, mobile, portable indoor)

- Type of receiving antenna

- Elevation angle

- Modulation system

- Type of delivery system (terrestrial, hybrid, satellite)

The methodology for calculation of the link margin is

Step 1: Frequency band

Step 2: Determination of satellite communication parameters

Step 3: Determination of receiver G/T

Step 4: Determine uplink budget to find out Uplink C/N

Step 5: Determine downlink power and noise budget for downlink $\mathrm{C} / \mathrm{N}$

Step 6: Propagation condition and uplink and downlink unavailability estimation.

Step 7: Calculate $E_{b} / N_{0}$

Step 8: Determine Link Margin

\subsubsection{Case-1: GEO Mission:}

The main parameters in GEO satellite are orbit (circular/equatorial), satellite height, round trip delay, satellite noise temperature, $\mathrm{Tx} / \mathrm{Rx}$ gain, satellite EIRP, polarization, antenna efficiency, reflector size, $3 \mathrm{~dB}$ beam width. Round trip delay is around $500 \mathrm{~ms}$ and noise temperature is around $600 \mathrm{~K}$. Radio link in GEO mission consists of transfer and on-orbit operations. Antennas employed in uplink for transfer orbit is having $11 \mathrm{~m}$ diameter whereas for on-orbit operations $7.2 \mathrm{~m}$ antenna is employed. Correspondingly, EIRP changes from $85 \mathrm{dBW}$ to $80 \mathrm{dBW}$ due to change in antenna diameter. The Link margin for command and tracking is calculated using link margin whereas $E_{b} / N_{0}$ is used for calculating link margin for telemetry data. Ka-band (uplink: $27.5-30.0 \mathrm{GHz}$; downlink: $17.8-20.2 \mathrm{GHz}$ ) is the band permitted by Federal Communications Commission (FCC) for operating service links and the gateway terminal links on a secondary non-interference basis to Teledesic. Transmitting and receiving frequencies are kept different so as to avoid all the earth stations/VSATs receiving their own frequencies.

The modulation scheme employed in the Indian GEO mission is $\mathrm{PCM} / \mathrm{PSK} / \mathrm{FM}$. Receiver processing gain in case of FM receiver is denoted as FM improvement and represented as,

$$
\left(\frac{S}{N}\right)_{\text {out }}=\left(\frac{C}{N}\right)_{\text {in }}+F M_{\text {imp }}
$$

\subsection{DOWNLINK ANALYSIS FOR TELEMETRY}

As known that antenna gain will quadruple with the doubling of frequency or antenna diameter (Eq.7(a)), but in the present study it is calculated keeping the same diameter as per Ku-band. The Table. 5 details the various parameters at different frequency bands and typical values for the calculation of the link margin. Implementation loss takes into account losses due to distortion, intermodulation, phase noise and other degradation introduced by receiver and transmitter.

\subsection{DEEP SPACE NETWORK}

The main characteristics and challenges in deep space communications are: Link interruption due to moving nodes, very long end-to-end delay which typically varies from 8.5 to $40 \mathrm{~min}$, high link error rate usually in order of $10^{-1}$, asymmetric bandwidth typically in order of 1000:1. Taking a case study of mission having at a distance of $R=406700 \mathrm{~km}$, the link margin for downlink analysis can be calculated as per Table.6 for S-band $(2.23 \mathrm{GHz})$ and $\mathrm{X}$-band $(8.4 \mathrm{GHz})$.

Table.5. Link Margin calculation for telemetry data at various frequency bands

\begin{tabular}{|c|c|c|c|c|c|c|}
\hline Parameters & Unit & S-band & $\begin{array}{c}\text { C- } \\
\text { band }\end{array}$ & $\begin{array}{c}\text { Ku- } \\
\text { band }\end{array}$ & X-band & $\begin{array}{c}\text { Ka- } \\
\text { band }\end{array}$ \\
\hline Orbit & $\mathrm{Kms}$ & 500 & 35800 & 35800 & 608 & 35800 \\
\hline Elevation & degree & 2 & 2 & 5 & 5 & 5 \\
\hline Transmitter O/P & $\mathrm{dBW}$ & -10 & 5.0 & -6.0 & & \\
\hline On board Losses & $\mathrm{dB}$ & & -4.0 & -5.0 & & -6.0 \\
\hline $\begin{array}{c}\text { Onboard Antenna } \\
\text { Gain }\end{array}$ & $\mathrm{dBi}$ & -10 & -10.0 & 25.0 & & 30 \\
\hline Polarisation Loss & $\mathrm{dB}$ & -3 & 0.0 & 0.0 & & -2.0 \\
\hline $\begin{array}{l}\text { EIRP }(\mathrm{dBW}) \\
{[\text { S.No } 2+3+4]}\end{array}$ & $\mathrm{dBW}$ & -23 & -9.0 & -14 & 25.0 & 17 \\
\hline Path Loss Eq.(3) & $\mathrm{dB}$ & -167.021 & -197.2 & -205.9 & -178.1 & -209 \\
\hline $\begin{array}{l}\text { Rain Attenuation/ } \\
\text { Misc Losses }\end{array}$ & $\mathrm{dB}$ & -1.0 & 0.0 & -6.0 & -2.0 & -14.0 \\
\hline $\begin{array}{c}\text { G/T of ground } \\
\text { station }\end{array}$ & $\mathrm{dB} / \mathrm{K}$ & 19.5 & 32.2 & 35.0 & 31 & 26 \\
\hline $\begin{array}{c}\text { Received CNDR } \\
{\left[\begin{array}{l}\text { S.No } 5+6+7+ \\
8+228.6]\end{array}\right.} \\
\end{array}$ & $\mathrm{dB}-\mathrm{Hz}$ & 57.079 & 54.6 & 65.7 & 104.5 & 63.6 \\
\hline Modulation Loss & $\mathrm{dB}$ & & -8.1 & -8.1 & - & -8.1 \\
\hline $\begin{array}{l}\text { Data rate with } \\
2 \mathrm{Kbps} \text { data } \\
\text { rate/320Mbps }\end{array}$ & $\begin{array}{c}\mathrm{dBHz} \\
\mathrm{Eq} .(12 \\
)\end{array}$ & $\begin{array}{c}-42 \\
(16 \mathrm{kbps})\end{array}$ & $\begin{array}{c}-33 \\
(2 \mathrm{kbps} \\
)\end{array}$ & -33 & -85.05 & $\begin{array}{c}-33 \\
(2 \mathrm{kbps} \\
)\end{array}$ \\
\hline $\begin{array}{c}\mathrm{E}_{\mathrm{b}} / \mathrm{N}_{0}[\mathrm{~S} . \mathrm{No} 9+ \\
10+11]\end{array}$ & & 15.079 & 11.5 & 22.6 & 19.44 & 22.5 \\
\hline $\begin{array}{l}\text { Implementation } \\
\text { Loss }\end{array}$ & $\mathrm{dB}$ & -2.0 & & & $\begin{array}{c}-[3.2+1.2 \\
(\text { pol } \\
\text { degrade })]\end{array}$ & -2.0 \\
\hline $\begin{array}{c}E_{b} / N_{0} \text { required } \\
\text { for BER of } 1 \mathrm{E}-6\end{array}$ & $\mathrm{~dB}$ & 10.8 & 11 & 5 & 10.8 & 5 \\
\hline Coding gain & $\mathrm{dB}$ & 5 & & & & \\
\hline $\begin{array}{c}\text { Margin [S.No } \\
12+13-14]\end{array}$ & $\mathrm{dB}$ & 7.72 & 0.5 & 17.6 & 4.24 & 15.5 \\
\hline
\end{tabular}


Table.6. Link Margin calculation for telemetry data at various frequency bands

\begin{tabular}{|c|c|c|c|c|}
\hline Parameters & Unit & $\begin{array}{c}\text { S - } \\
\text { Band } \\
(\mathrm{TM})\end{array}$ & $\begin{array}{c}\text { X -band } \\
\text { (Data } \\
\text { Tx) }\end{array}$ & Remarks \\
\hline Orbit (R) & $\mathrm{Kms}$ & 406700 & 406700 & \\
\hline Transmitter O/P & dBW & 7 & 7 & \\
\hline On board Losses & $\mathrm{dB}$ & -6 & & \\
\hline Onboard Antenna Gain & $\mathrm{dBi}$ & -2 & $\ldots$ & \\
\hline Elevation & degree & .. & 5 & \\
\hline Polarisation Loss & $\mathrm{dB}$ & $\ldots$ & 0.2 & \\
\hline $\mathrm{EIRP}(\mathrm{dBW})$ & $\mathrm{dBW}$ & -1 & 37.0 & $\begin{array}{l}\text { S.No } 1+2+ \\
3-5\end{array}$ \\
\hline Path Loss Eq.(3) & $\mathrm{dB}$ & 211.54 & -223.2 & $\begin{array}{c}\text { S.No } 7=8+ \\
9\end{array}$ \\
\hline Spread Loss & $\mathrm{dBm}^{2}$ & 183.2 & $\ldots \ldots$ & $\pi R^{2} / 4$ \\
\hline Aperture Factor & $\mathrm{dBm}^{2}$ & 28.4 & $\ldots \ldots$ & $\lambda^{2} / 4 \pi$ \\
\hline $\begin{array}{c}\text { Antenna Pointing } \\
\text { Losses }\end{array}$ & $\mathrm{dB}$ & 0.2 & 0.2 & \\
\hline G/T of ground station & $\mathrm{dB} / \mathrm{K}$ & 25.6 & 36.0 & \\
\hline Received CNDR & $\mathrm{dB}-\mathrm{Hz}$ & 41.46 & 78.2 & $\begin{array}{c}\text { S.No } 6-8- \\
9-10-5+ \\
11+228.6\end{array}$ \\
\hline Modulation Loss & $\mathrm{dB}$ & 5.6 & - & \\
\hline $\begin{array}{c}\text { Data Rate with } 1 \mathrm{kbps} \\
\text { data rate/8.4Mbps }\end{array}$ & \begin{tabular}{|c|}
$\mathrm{dB}-\mathrm{Hz}$ \\
$(\mathrm{Eq} \cdot(12))$
\end{tabular} & 30 & 69.20 & \\
\hline Available $E_{b} / N_{0}$ & & 5.8 & 9.0 & $\begin{array}{c}\text { S.No } 9-10 \\
-11\end{array}$ \\
\hline Implementation Loss & $\mathrm{dB}$ & 2.0 & 2.0 & \\
\hline $\begin{array}{l}E_{b} / N_{0} \text { required for BER } \\
\text { of } 1 \mathrm{e}^{-5}\end{array}$ & $\mathrm{~dB}$ & 2.3 & 2.8 & \\
\hline Coding gain & $\mathrm{dB}$ &.. & & \\
\hline Margin & $\mathrm{dB}$ & 1.5 & 4.20 & $\begin{array}{c}\text { S.No } 12-13 \\
-14\end{array}$ \\
\hline
\end{tabular}

\section{CONCLUSION}

Link analysis and various loss mechanism associated with as well as propagation path statistics is detailed in this article. Cases of both LEO and GEO systems are taken for studying various propagation effects. At $\mathrm{C}$-band and $\mathrm{Ku}$-band simultaneously fading of uplink and downlink is not encountered while at Ka-band this effect is to be considered also. Attenuation rate increases rapidly at millimetric wave frequencies due to atmospheric effects such as absorption and precipitation. C-band communication requires less link margin compare to Ka-band because atmospheric losses increases with frequency and are variable. In the coexistence scenarios of GEO and NGEO networks, in-line interference can cause link break whenever an NGEO satellite passes through a line of sight path between and earth station and a GEO satellite. Many techniques to avoid this phenomena such as spot turn off, inter and intra orbital plane frequency division can avoid or minimize the co-channel interference. Capacity enhancement provided by millimetric system is necessary because electronically earth orbit is becoming crowded and to overcome this phenomenon the various methodologies adopted are- increasing directionality of the transmitted beams from the satellite to reduce interference, increase amount of useful information and increasing number of frequency bands. The most effective way is by using high frequency which will enhance bandwidth, remove interference, directionality of antenna beam resulting in stronger receive signal having closer spacing to accommodate more satellites. This article will help radio engineers to predict the link margin easily with the defined approach at various frequency bands.

\section{REFERENCES}

[1] D.M. Pozar, "Microwave Engineering", John Wiley and Sons, 2010.

[2] S.K. Sharama, S. Chatzintos and B. Otersten, "In-line Interference Mitigation Techniques for Spectral Coexistence of GEO and NGEO Satellites", International Journal of Satellite Communications, Vol. 34, No. 1, pp. 11-39, 2016.

[3] V Sambasiva Rao, "Extend LEO Downlinks with GEO Satellites", Available at: http://www.mwrf.com/datasheet/extend-leo-downlinksgeo-satellites-pdf-download.

[4] Aderemi A. Atayero, Matthew K. Luka and Adeyemi A. Alatishe, "Satellite Link Design: A Tutorial", International Journal of Electrical and Computer Sciences, Vol. 11, No. 4, pp. 1-6, 2011.

[5] C. Haslett, "Essentials of Radio Wave Propogation", Cambridge Wireless Essentials Series, 2008.

[6] R. Ashiya, "A Regional Satellite System for Mobile Communications", Proceedings of IEEE International Conference on Personal Wireless Communications, pp. 142 146, 1994.

[7] K.N. Madhavan, D. John, A. Bhaskaranarayana,T.S. Narayan, M. Rathnakumar, V.K. Lakshmeesha and S. Pal, "TT\&C Transponder for INSAT-2 Series Satellites", IETE Technical Review, Vol. 11, No. 5, pp. 291-296, 1994.

[8] W.S. Cheung and F.H. Leuvien, "Microwave Made Simple", Artech House, 1995.

[9] T.C. Barbosa,R.L. Moreno,T.C. Pereira and L.H.C. Ferreira, "FPGA Implementation of a Reed Solomon Codec for OTNG.709 Standard with reduced Decoder Area", Proceedings of $6^{\text {th }}$ International Conference on Wireless Communications Networking and Mobile Computing, pp. 14, 2010.

[10] Daniel Minoli, “Innovations in Satellite Communications Technology", Wiley, 2015.

[11] L.S Chuan, S.R-Tian andY.P Hon, "Ka-Band Satellite Communications Design Analysis and Optimisation", DSTA Horizons, pp. 70-78, 2015.

[12] S. Dey, D.K. Mohapatra and S.D.R.P. Archana, "An Approach to calculate the Performance and Link Budget of LEO Satellite (Iridium) for Communication Operated at frequency Range (1650-1550)MHz", International Journal of Latest Trends in Engineering and Technology, Vol. 4, No. 4, pp. 96-103, 2014. 